\title{
Synthesis and Evaluation of L-glutamide Derived Carbonyl Group Containing RP-HPLC Phase for Polycyclic Aromatic Hydrocarbon (PAH) Separation
}

\author{
A. A. Rana*a, M. Kamruzzamana, and M. Takafuji ${ }^{\mathrm{b}}$ \\ ${ }^{a}$ Department of Applied Chemistry \& Chemical Engineering, University of Dhaka, Dhaka-1000, Bangladesh \\ and ${ }^{b}$ Department of Applied Chemistry \& Biochemistry, Kumamoto University, \\ 2-39-1 Kurokami, Kumamoto 860-8555, Japan
}

\begin{abstract}
A new L-glutamide derived internal $\mathrm{C}=\mathrm{O}$ group containing HPLC stationary phase was synthesized. The L-glutamide lipid was immobilized on to silica surface through aminopropyltrimethoxysilane (APS) linker. The detailed chemical structure of the compounds leading to the final lipid was characterized by melting point measurement, FTIR, ${ }^{1} \mathrm{H}$ NMR and elemental analysis in each step. Chromatographic properties of silica immobilized N-(pyrinylbutyric) L-glutamic acid, Sil-PBG, were evaluated under reversed phase conditions by separation of different polycyclic aromatic hydrocarbons (PAHs) and the results were then compared with monomeric octadecylated silica stationary phase (ODS). Chromatographic evaluation of the new phase showed promising results for the separation of PAHs.
\end{abstract}

Key words: L-glutamide lipid, RP-HPLC, Enhance selectivity, Polycyclic aromatic hydrocarbons

\section{Introduction}

Chemical modification of silica packing materials remains a popular approach for achieving novel solute selectivity in high performance liquid chromatography (HPLC) (Jinno et al, 1987; Jinno et al, 1992; Saito et al, 1994 and Kirkland et al, 1989). Ihara et. al. have proposed that self-assembled systems such as lipid membrane aggregates can provide a highly ordered microenvironment leading to unique hostguest chemistry exceeding the functions of the original lipid. Earlier we reported about the use of poly- (octadecyl acrylate)-grafted silica $\left(\mathrm{Sil}_{-}-\mathrm{ODA}_{\mathrm{n}}\right)$, a lipid membrane analogue, as stationary phase for RP-HPLC (Hirayama et al 1992). Sil-ODA $_{n}$ showed unique separation behaviors with orderedto-disordered phase transitions of long alkyl chains. In particular, extremely high selectivity toward polycyclic aromatic hydrocarbons (PAHs) was observed in the ordered (crystalline) state (Fukumoto et al, 1994; Chowdhury et al, 2002; Chowdhury et al, 2000 and Ihara et al, 1996). We have found that aligned carbonyl groups in Sil-ODA $\mathrm{n}$ are effective for PAH selectivity. Previously we have reported that carbonyl $\pi-\pi$ interactions are also very effective in RP-HPLC application even in the disordered state (Ashequl et al, 2009). On the other hand, it is well known that the lipophilic L-glutamic acid-derived systems with three amide bonds work as self-assembling materials. Therefore, this system

\footnotetext{
* Corresponding author: E-mail: rana_3786@yahoo.com,
}

may have potential applications for various fields such as catalysis, sensor technology, materials science, and separation science (Takafuji et al, 2004). Our group has successfully implemented this system in the separation science especially in the chromatographic application. It was reported that amide bonding in the L-glutamic acid derivatives plays an important role in self-assembling and the embedded carbonyl groups in the system which are aligned, enhance the selectivity through $\pi-\pi$ interaction (Mizanur et al, 2005). In this article, we try to develop a silica immobilized new stationary phase based on L-glutamic acid derivative, $\mathrm{N}$ (pyrenylbutyric) glutamide (Sil-PBG) and compare the separation of polycyclic aromatic hydrocarbons (PAH's) with commercial ODS. This new phase has no alkyl chain rather it possesses functional carbonyl group and one pyrene group in the system which may act as additional $\pi-\pi$ interactive source. However, in this stationary phase we reduce the number of amide bonding to concentrate on the effect of carbonyl group in the separation process.

\section{Materials and Methods}

Starting from L-glutamic acid all chemicals for the synthesis of lipid was purchased from Wako Pure Organic Chemical 
Industries, Sigma Aldrich, Tokyo Kasei Kogyo Co. Ltd. (Tokyo, Japan) and Nacalai Tesque Inc. (Kyoto, Japan), respectively, and used as received. 3-Aminopropy ltrimethoxysilane (APS) was purchased from Azmax Co. (Chiba, Japan). Porous silica particles (YMC-GEL) were purchased from YMC Co. Ltd. (Kyoto, Japan) whose average diameter; pore size and surface area are $5 \mu \mathrm{m}, 12.0 \mathrm{~nm}$ and $339 \mathrm{~m}^{2} \mathrm{~g}^{-1}$, respectively. Sil-PBG was synthesized and packed into stainless steel column for RP-HPLC. The monomeric ODS column (Intersil ODS 3, column size 250 $\mathrm{mm} \times 4.6 \mathrm{~mm}$ i.d.) with particle size $5.5 \mu \mathrm{m}$ and surface area of $450 \mathrm{~m}^{2} \mathrm{~g}^{-1}$ ) was purchased from G.L. Science (Tokyo, Japan).

\section{Preparation of L-glutamide derived lipid}

The synthesis scheme of lipid N (pyrenylbutyric) L-glumatic acid and the immobilization process of the membrane onto silica were shown in scheme 1 . The detailed chemical struc ture of the compounds leading to the final product were characterized by melting point measurement, FTIR, ${ }^{1} \mathrm{H}$ NMR and elemental analysis in each step.

\section{Preparation of $\alpha, \gamma$ - glutamic acid benzyl ester (1)}

L-glutamic acid (33.98 mmol), benzyl alcohol (118.92 $\mathrm{mmol})$ and p-toluene sulphonic acid $(51 \mathrm{mmol})$ were dissolved in $200 \mathrm{ml}$ benzene. The reaction was conducted using dean stark equipment. After the first drop of condensate, the reaction was continued for 5 hrs. The solution was then cooled to room temperature and further cooled to $0^{\circ} \mathrm{C}$ in the freeze. The solution was carefully filtered in vacuo and washed several times with benzene. Finally the product was washed with diethyl ether and dried under vacuum to remove the solvent yielding white product.
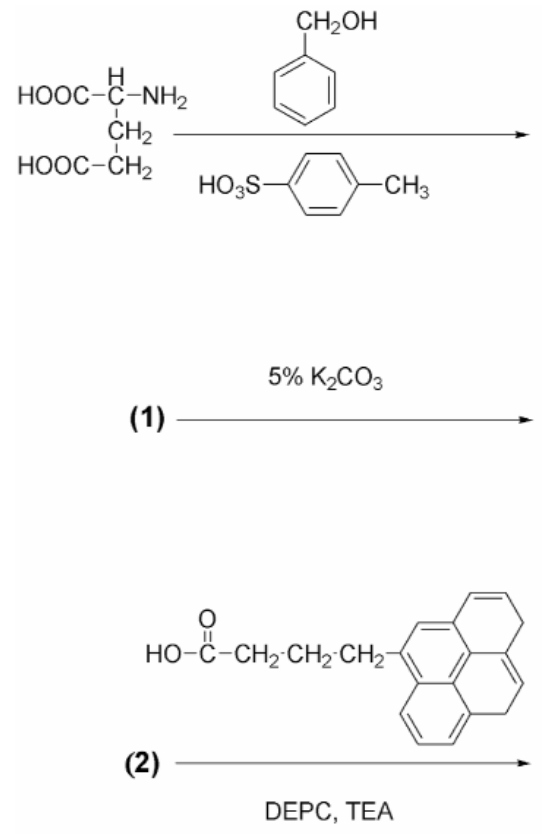

(3) $\longrightarrow$

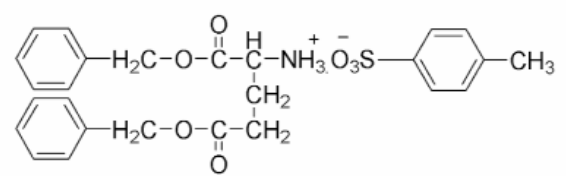

(1)

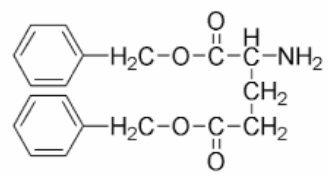

(2)

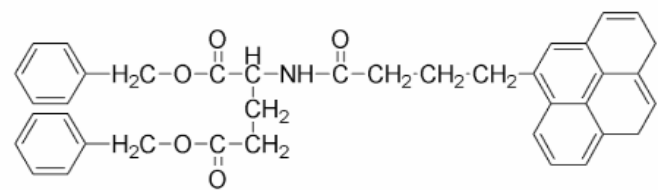

(3)

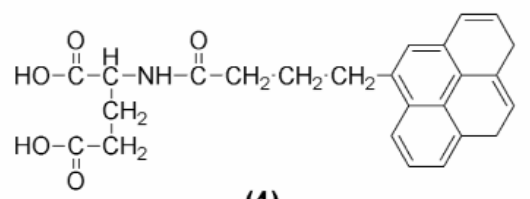

(4)

Scheme 1: Reaction scheme for the synthesis of L-glutamide lipid membrane 
$m . p: 144-145^{\circ} \mathrm{C}$.

FTIR data: $v_{(\max )} / \mathrm{cm}: 1710$ (ester $\left.\mathrm{C}=\mathrm{O}\right), 2851,2917$ (aliphatic C-H)

${ }^{1} \mathrm{H}$ NMR data $\left(\mathrm{CD}_{3} \mathrm{OD}\right): \delta$ 2.15-2.22 $(\mathrm{m}, \quad 2 \mathrm{H}$, $\mathrm{CHCH}_{2} \mathrm{CH}_{2} \mathrm{CO}$ ), 2.36 (s, 3H, $\left.\mathrm{C}_{6} \mathrm{H}_{5} \mathrm{CH}_{3}\right), 2.50-2.60(\mathrm{~m}, 2 \mathrm{H}$, $\left.\mathrm{CHCH}_{2} \mathrm{CH}_{2} \mathrm{CO}\right), 4.13-4.17\left(\mathrm{t}, 1 \mathrm{H}, \mathrm{CH}^{*}\right), 5.12$ and $5.25(\mathrm{~s}$, $\left.4 \mathrm{H}, \mathrm{C}_{6} \mathrm{H}_{5} \mathrm{CH}_{2}\right), 7.36\left(\mathrm{~s}, 10 \mathrm{H}, \mathrm{CH}_{2} \mathrm{C}_{6} \mathrm{H}_{5}\right.$ )

Table I: Elemental analysis data of compound (1)

\begin{tabular}{l|c|c|c|c}
\hline & $\mathrm{C} \%$ & $\mathrm{H} \%$ & $\mathrm{~N} \%$ & $\mathrm{C} / \mathrm{N}$ \\
\hline Anal (calculated) & 62.53 & 5.81 & 2.81 & 22.3 \\
Anal (found) & 61.87 & 5.96 & 2.70 & 22.9 \\
\hline
\end{tabular}

\section{Preparation of dibenzyl L-glutaminate (2)}

Dissolve about $15.5 \mathrm{~g}$ of compound (1) in $90 \mathrm{ml}$ chloroform and transfer the chloroform solution in separating funnel. Wash the organic solution 2-3 times with $5 \% \mathrm{~K}_{2} \mathrm{CO}_{3}$ solution. Collect the aqueous layer and check pH which should be above 10. Then wash the organic layer with Di-ionized water. Collect the organic layer and dried over $\mathrm{Na}_{2} \mathrm{SO}_{4}$. Finally the compound was dried in vacuum.

${ }^{1} \mathrm{H}$ NMR data (in $\left.\mathrm{CDCl}_{3}\right): \delta$ 1.85-2.20 (m, 2H, $\mathrm{CHCH}_{2} \mathrm{CH}_{2} \mathrm{CO}$ ), 2.47-2.56 (t, $2 \mathrm{H}, \mathrm{CHCH}_{2} \mathrm{CH}_{2} \mathrm{CO}$ ), 3.48$3.51\left(\mathrm{~m}, 1 \mathrm{H} \mathrm{CH}^{*}\right), 5.18$ and $5.28\left(\mathrm{~s}, 4 \mathrm{H}, \mathrm{C}_{6} \mathrm{H}_{5} \mathrm{CH}_{2}\right), 7.33(\mathrm{~s}$, $\left.10 \mathrm{H}, \mathrm{C}_{6} \mathrm{H}_{5} \mathrm{CH}_{2}\right)$

Preparation of N (pyrenylbutyric) - L - glutamic benzylester (3)

Compound 2 (13.84 mmol) along with pyrenebutyric acid (13.84 mmol), triehyl amine (41.52 mmol) and DEPC (20.8 mmol) were dissolved in $350 \mathrm{ml} \mathrm{THF}$. The reaction was conducted at $0^{\circ} \mathrm{C}$ and continued for 1 day. The solvent was evaporated and the resulting product was washed thoroughly with $0.2 \mathrm{~N} \mathrm{HCl}, 5 \% \mathrm{Na}_{2} \mathrm{CO}_{3}$ and DI water. Then the product is dried over sodium sulphate. This liquid mass was then recrystallized from diethyl ether to give a pale yellow solid powder.

m.p: $163-164^{\circ} \mathrm{C}$

FTIR data: $v_{\max } / \mathrm{cm}: 1540$ (amide $\mathrm{N}-\mathrm{H}$ ), 1646 (amide $\mathrm{C}=\mathrm{O}$ ), 1731 (ester $\mathrm{C}=\mathrm{O}$ ), 2875(aliphatic $\mathrm{C}-\mathrm{H}$ ), 2943, 3034(aromatic C-H) and 3302(amide N-H)
${ }^{1} \mathrm{H}$ NMR data $\left(\mathrm{CDCl}_{3}\right): \delta 2.18-2.45\left(\mathrm{~m}, 4 \mathrm{H}, \mathrm{CHCH}_{2} \mathrm{CH}_{2} \mathrm{CO}\right.$; $\left.\mathrm{CH}_{2} \mathrm{C}_{10} \mathrm{H}_{9}\right), 3.36-3.38$ (t, 2H, $\left.\mathrm{CHCH}_{2} \mathrm{CH}_{2} \mathrm{CO}\right), 4.65-4.76$ $\left(\mathrm{m},{ }^{1} \mathrm{H}, \mathrm{CH}^{*}\right), 5.03$ and $5.15\left(\mathrm{~s}, 4 \mathrm{H}, \mathrm{C}_{6} \mathrm{H}_{5} \mathrm{CH}_{2}\right), 7.31(\mathrm{~s}, 10 \mathrm{H}$, $\left.\mathrm{C}_{6} \mathrm{H}_{5} \mathrm{CH}_{2}\right)$ and 7.84-8.30 (m, 9H, $\left.\mathrm{C}_{16} \mathrm{H}_{9}\right)$

Table II: Elemental analysis data of compound (3)

\begin{tabular}{l|c|c|c|c}
\hline & $\mathrm{C} \%$ & $\mathrm{H} \%$ & $\mathrm{~N} \%$ & $\mathrm{C} / \mathrm{N}$ \\
\hline Anal (calculated) & 78.40 & 5.86 & 2.35 & 33.36 \\
Anal (found) & 74.87 & 5.94 & 2.39 & 31.33 \\
\hline
\end{tabular}

\section{Preparation of N (pyrenylbutyric) L-glutamic acid (4)}

Compound (3) and $100 \mathrm{ml}$ ethanol were taken in a three necked round bottom flask and heated until the reaction temperature reached about $60^{\circ} \mathrm{C}$. Pd-carbon $(500 \mathrm{mg})$ catalyst was added in the solution at $60^{\circ} \mathrm{C}$ and thus the hydrogenation reaction was continued and constantly monitored with the help of IR spectrum. The hydrogenation was completed after 2 hrs. After hydrogenation, the solvent was evaporated and the solid mass was washed properly. The product was then recrystallized from diethyl ether. The FTIR and ${ }^{1} \mathrm{H}$ NMR spectra of the lipid were shown in figure 1 and 2 respectively.

$m \cdot p: 156-158^{\circ} \mathrm{C}$

FTIR data: $v_{\max } / \mathrm{cm}: 1541,1635,1721,2940,3040,3354$

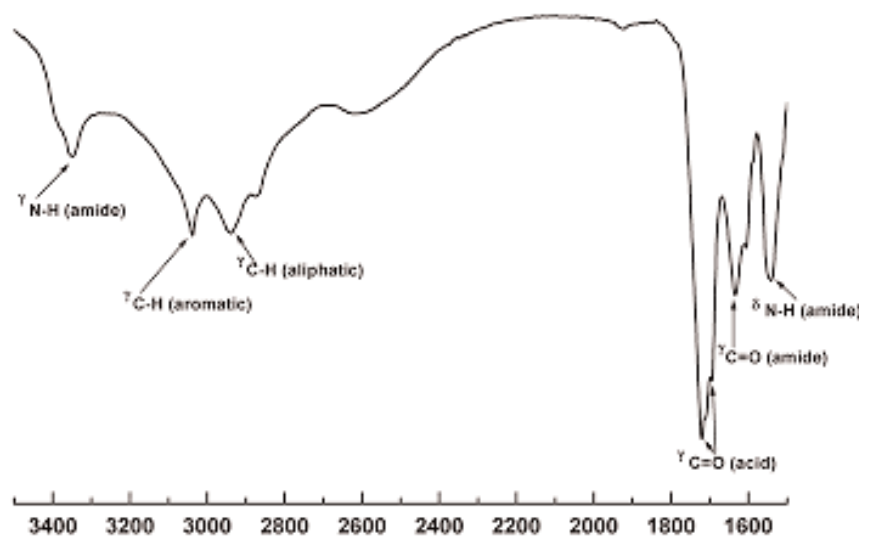

Fig.1: FTIR spectrum of N (pyrenylbutyric) L-glutamic acid

${ }^{1} \mathrm{H}$ NMR data $\left(\mathrm{CD}_{3} \mathrm{OD}\right): \delta$ 1.16-1.19 (m, 2H, $\left.\mathrm{COCH}_{2} \mathrm{CH}_{2}\right)$, 1.9-2.02 $\left(\mathrm{C}_{16} \mathrm{H}_{9} \mathrm{CH}_{2}\right), 2.12-2.28\left(\mathrm{~m}, 2 \mathrm{H}, \mathrm{COCHCH}_{2}\right), 2.39$ $2.57\left(\mathrm{~m}, 4 \mathrm{H}, \mathrm{NHCOCH}_{2} ; \mathrm{CH}_{2} \mathrm{CH}_{2} \mathrm{CO}\right), 4.47-4.53(\mathrm{t}, 1 \mathrm{H}$, $\left.\mathrm{CH}^{*}\right)$, 7.9-8.36 (m, 9H, $\left.\mathrm{CH}_{2} \mathrm{C}_{16} \mathrm{H}_{9}\right)$. 


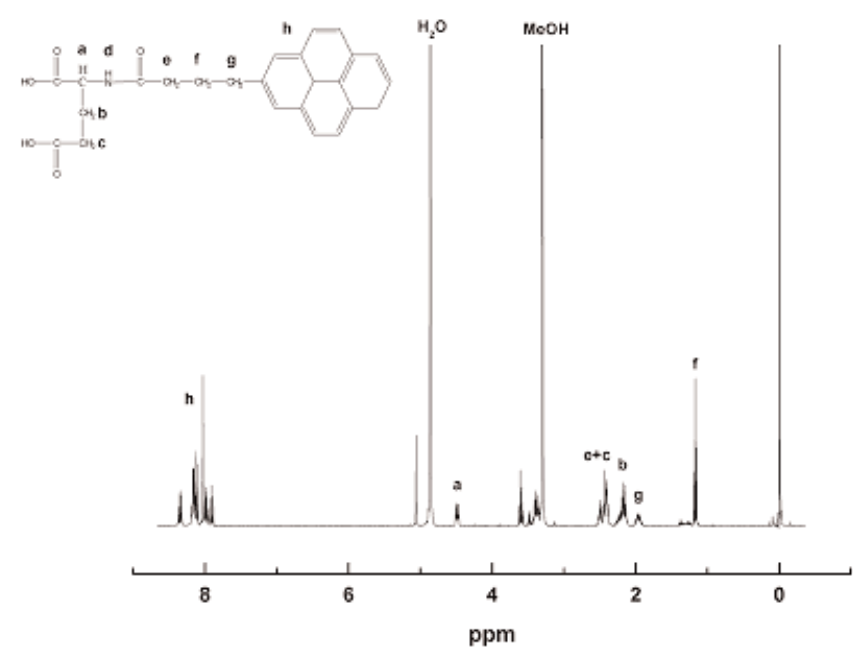

Fig. 2: ${ }^{1} \mathrm{H}$ NMR spectrum of $\mathbf{N}$ (pyrenylbutyric) L-glutamic acid

Table III: Elemental analysis data of $\mathbf{N}$ (pyrenylbutyric) L-glutamic acid

\begin{tabular}{l|c|c|c|c}
\hline & $\mathrm{C} \%$ & $\mathrm{H} \%$ & $\mathrm{~N} \%$ & $\mathrm{C} / \mathrm{N}$ \\
\hline Anal (calculated) & 71.94 & 5.52 & 3.36 & 21.4 \\
Anal (found) & 70.36 & 5.83 & 3.52 & 20.0 \\
\hline
\end{tabular}

\section{Immobilization of lipid membrane on to silica}

3-aminopropyltrimethoxysilane grafted silica (5) Sil-APS was prepared by refluxing porous silica gel $(3.0 \mathrm{~g})$ and 1.5 $\mathrm{mL}$ of APS in toluene for $24 \mathrm{hrs}$. After successive washing with toluene, ethanol and di ethyl ether the particles were dried in vacuo. The dried particles were characterized by elemental analysis and Drift-IR. Sil-APS (5) (3.0 g) and lipid (4) $(3.0 \mathrm{~g})$ were taken in a $100 \mathrm{~mL}$ dry THF and stirred.

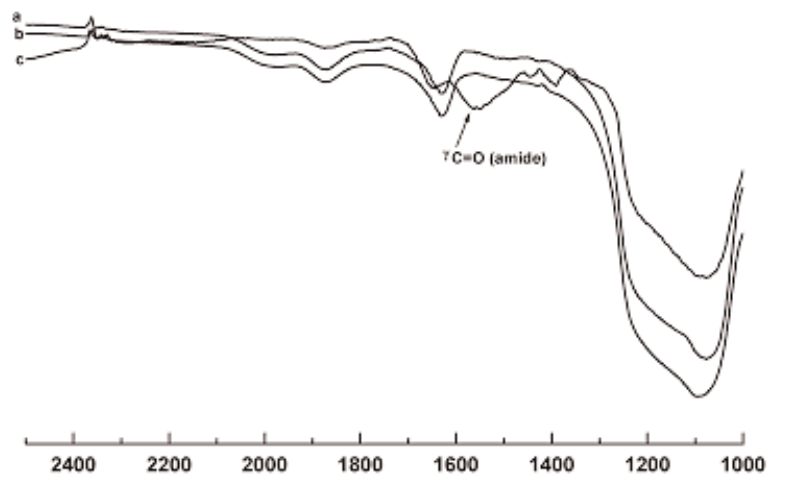

Fig. 3: Drift-IR spectrum of bare silica (a), Sil-APS (b) and Sil-PBG (c)
DEPC (1.5 g) and TEA (1.1 g) were added to the solution and stirred at room temperature. After stirring for $24 \mathrm{hrs}$, the silica grafted particles were washed with THF and chloroform several times to remove unconverted lipid molecule and dried in vacuum. Introduction of carbonyl bonding confirmed the grafting of lipid onto silica surface (Figure 3).

Table IV: Elemental analysis data of Sil-APS and SilPBG

\begin{tabular}{l|c|c|c}
\hline & $\mathrm{C} \%$ & $\mathrm{H} \%$ & $\mathrm{~N} \%$ \\
\hline Sil-APS & 8.08 & 2.05 & 2.88 \\
Sil-PBG & 16.16 & 2.85 & 2.90 \\
\hline
\end{tabular}

\section{Results and Discussion}

\section{HPLC system}

The chromatographic system consists of a Gulliver PU-980 intelligent HPLC pump with a Rheodyne sample injector having $20 \mu \mathrm{L}$ loops. A Jasco multi-wavelength UV detector MD 2010 plus was used. The column temperature was maintained by using a column jacket with a circulator having a heating and a cooling system. A personal computer connected to the detector with Jasco-Borwin (Ver 1.5) software was used for system control and data analysis. As the sensitivity of UV detector is high, $5 \mu \mathrm{L}$ of sample solution was used for each injection. Chromatographic measurements were performed using HPLC grade methanol and water mixture (80:20) as mobile phase at a flow rate of 0.5 and $1.00 \mathrm{~mL}$ $\min ^{-1}$ respectively. The retention factor $(k)$ measurement was done under isocratic elution conditions. The separation factor $(\alpha)$ is the ratio of the retention factor of two solutes that are being analyzed. The retention time of $\mathrm{D}_{2} \mathrm{O}$ was used as the void volume $\left(t_{0}\right)$ marker (the absorption for $\mathrm{D}_{2} \mathrm{O}$ was measured at $400 \mathrm{~nm}$, which actually considered as injection shock). All data points were derived from at least triplicate measurements; with retention time $\left(t_{\mathrm{R}}\right)$ value varying $\pm 1 \%$. Water/1-octanol partition coefficient $(P)$ was measured by the retention studies with octadecylated silica, ODS (monomeric) (Inertsil ODS, i.d. $250 \mathrm{~mm} \times 4.6 \mathrm{~mm}$, GL Science, Tokyo, Japan): $\log P=3.579+4.207 \log k(\mathrm{r}) 0.999$ 997) (Shundo et al, 2005).

\section{Selectivity for PAHs}

The shape selectivity of a given stationary phase can be measured by the separation factor $(\alpha)$ value for pairs of test 
solutes having the same carbon number but different shape, such as triphenylene/o-terphenyl, chrysene/pyrene and trans-stilbene/ cis-stilbene. On a typical monomeric ODS phase, the separation factor for triphenylene/o-terphenyl ranges from 1.0 to 1.7 , while the value on a polymeric ODS phase lies between 2.0 and 2.7. Jinno et al.(1990) and Jinno et al, (1989) suggested that a separation factor $(\alpha)$ for triphenylene/o-terphenyl $\geq=2.0$ is an indication of significant solute planarity recognition. We have observed (as shown in Fig. 4) that, Sil-PBG (triphenylene/o-terphenyl = 3.6) possesses enhanced molecular planarity selectivity for PAHs than ODS. Other sets for planarity selectivity also provide better result than ODS which were given in Table V.

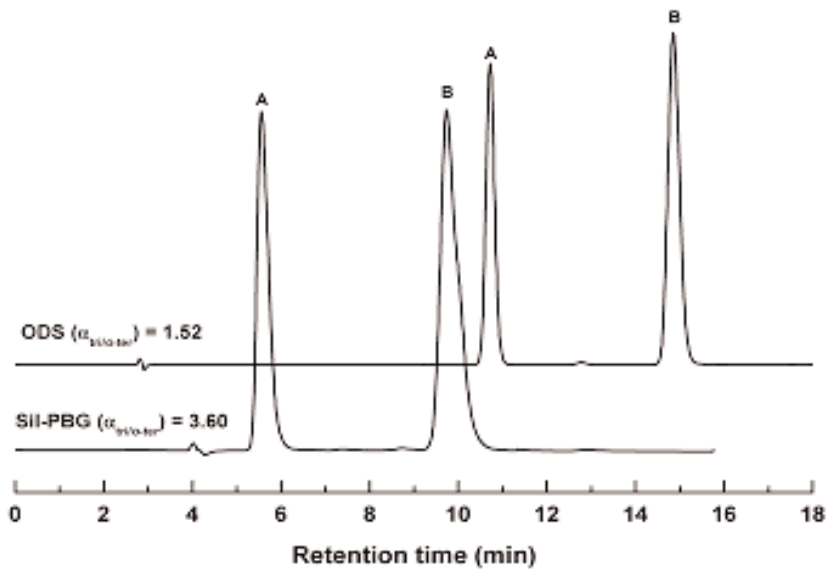

Fig. 4: Separation of $o$-Terphenyl (A) and Triphenylene (B) in Sil-PBG and ODS at $25^{\circ} \mathrm{C}$. mobile phase: methanol/water $(80 / 20)$, flow rate: $0.5 \mathrm{ml} / \mathrm{min}$ for Sil-PBG and $1.0 \mathrm{ml} / \mathrm{min}$ for ODS, UV detection: $254 \mathrm{~nm}$.

The uniqueness of Sil-PBG can also be found in the separation of terphenyl-isomers. Three terphenyl-isomers differ strongly in the extent of their non-planarity (Horak et al, 2004). The close proximity of the two $\pi$-electron clouds of the aryl groups in ortho-position provides a strong sterical hindrance that is amplified by the repulsion of the two aromatic $\pi$-electron clouds. Hence the $o$-terphenyl isomer possesses the highest deviation from planarity followed by $m$ terphenyl and $p$-terphenyl and similar was the retention order with Sil-PBG along with higher separation factor between the $p$-/o-terphenyls compared to the ODS reference column as shown in figure 5 .

Specificity of Sil-PBG was also observed in for structural isomers of four ring compounds. All of them are planar and

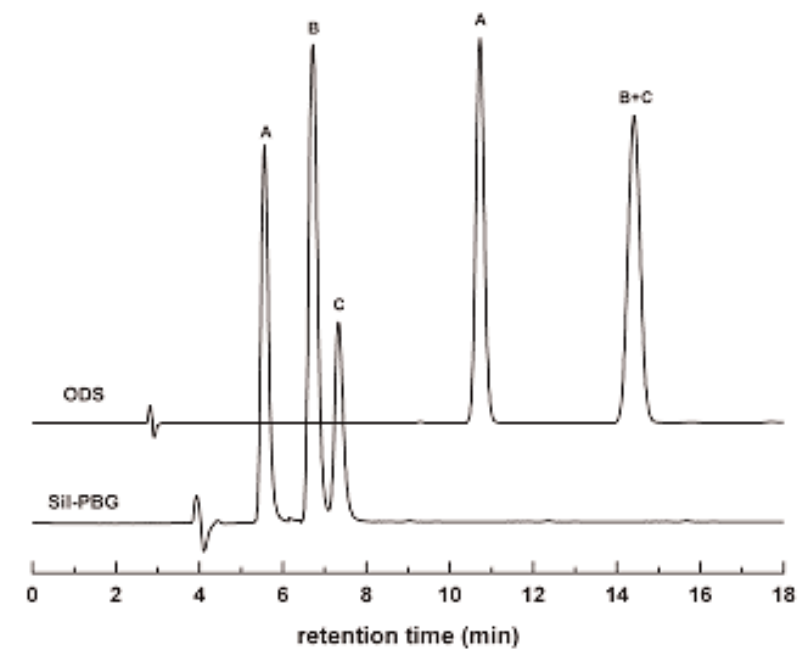

Fig. 5: Chromatograms of a mixture of terphenyl isomers with Sil-PBG and ODS. Mobile phase: methanol/water $(80 / 20)$, temperature: $25^{\circ} \mathrm{C}$ and UV detection: 254 nm., elutes: A) o-Terphenyl, B) $m$-Terphenyl and C) $p$-Terphenyl

contain equal number of carbon and $\pi$ electrons. Better selectivity was observed for Sil-PBG as compared with ODS (Table V).

The higher selectivity by Sil-PBG can be explained by $\pi-\pi$ contribution from the carbonyl groups and pyrene moiety present in the lipid membrane and the $\pi$-electrons present in the guest PAHs. Elution of $o$-terphenyl before anthracene indicates significant $\pi-\pi$ interaction in the Sil-PBG as in ODS the elution is in reverse order. We have reported previously that carbonyl groups in Sil-ODA $\mathrm{n}_{\mathrm{n}}$ are polarized to $\delta^{+}$ (carbon) and $\delta^{-}$(oxygen) (Ihara et al, 1999 and Ihara et al, 2005). These polarized atoms can work as an electrostatic source of $\pi-\pi$ interaction in which carbon atoms act as electron acceptors and interact with the $\pi$-electron containing guest molecules. The carbonyl groups in stationary phases interact with aromatic elutes through $\pi-\pi$ interactions that are stronger $\left(1.87 \mathrm{kcal} \mathrm{mol}^{-1}\right.$ in HCHO-benzene) than those of both $\mathrm{CH}-\pi\left(0.57 \mathrm{kcal} \mathrm{mol}^{-1}\right.$ in $\mathrm{CH}_{4}$-benzene) and benzene $\pi$-benzene $\pi$ ( $0.49 \mathrm{kcal} \mathrm{mol}^{-1}$ in the plane-to-plane stacking) (Sakaki et al, 1993) and the aligned carbonyl groups in Sil$\mathrm{ODA}_{n}$ are effective for enhancing higher selectivity toward PAHs. We believe that this theory is also applicable towards this stationary phase. However further investigation is required for the assessment of retention mechanism. 
Table V: retention $(k)$ and separation factor $(\alpha)$ of PAHs for Sil-PBG and ODS in RP-HPLC

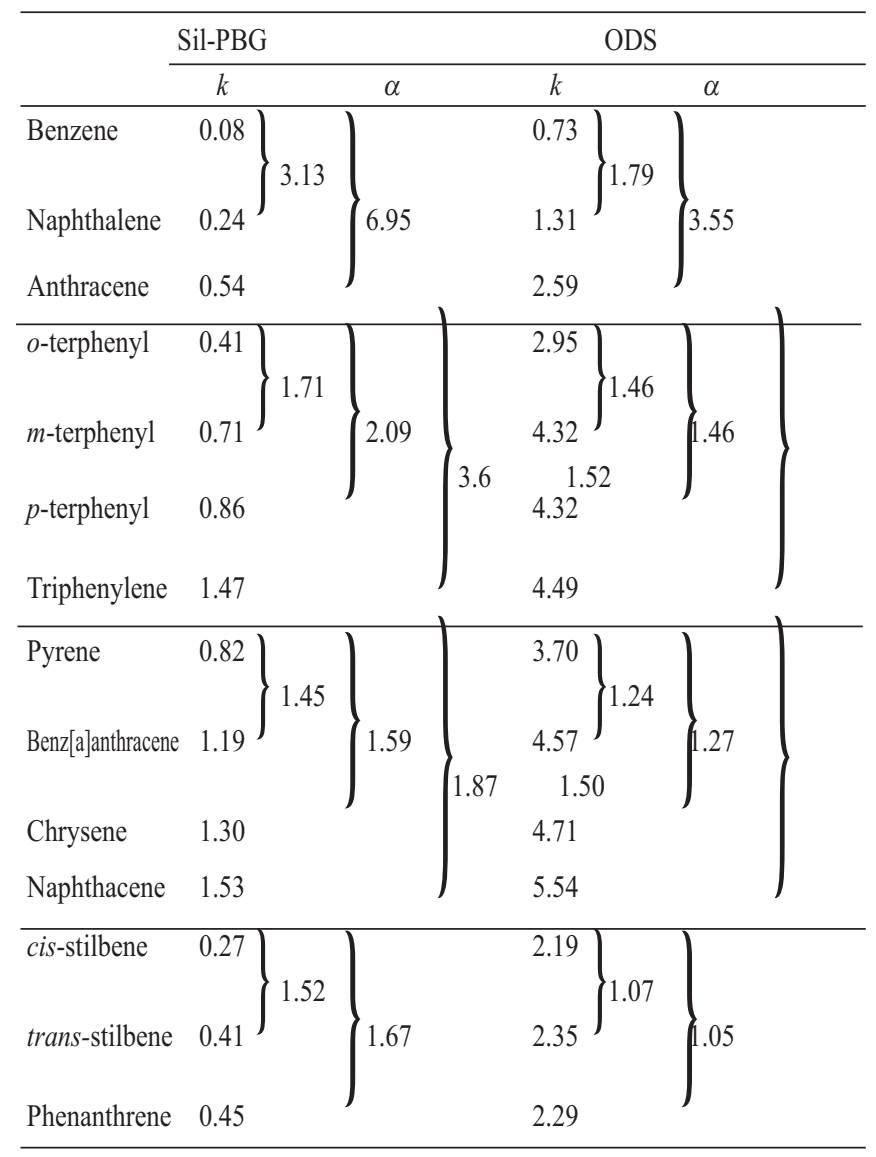

\section{Conclusion}

In conclusion, successful synthesis of a lipid membrane from L-glutamic acid derivative has been done. The lipid was grafted onto silica through aminopropyltrimethoxysilane linker. Chromatographic results showed that enhanced selectivity was observed for PAHs and aromatic positional isomers than ODS phase. The enhancement of selectivity for PAHs on Sil-PBG can be demonstrated from the contribution of $\pi-\pi$ interactions between the guest molecules and the carbonyl groups present in the lipid chain. Moreover, the presence of pyrene moiety further boosts its selectivity by $\pi-\pi$ interactions with guest molecules.

\section{References}

Ashequl. A., Rana Makoto Takafuji and Ihara H. (2009). J of Chromatogr. A; 1216: 7440-7445.

Chowdhury M. A. J., Boysen R. I., Ihara H. and Hearn M. T. W. (2002). J. Phys. Chem. B 106: 11936-11950.
Chowdhury M. A. J., Ihara H., Sagawa T. and Hirayama C. (2000). J. Chromatogr., A. 877: 71-85

Fukumoto T., Ihara H., Sakaki S., Shosenji H., Hirayama C. (1994). J. Chromatogr. A; 672: 237-241.

Hirayama C., Ihara H. and Mukai T. (1992). Macromolecules 25: 6375-6376.

Horak J. and Lindner W. (2004). J. Chromatogr, A 1043177.

Ihara H., Sagawa T., Goto Y. and Nagaoka S. (1999). Polymer 40: 2555.

Ihara H., Takafuji M., Sakurai T., Sagawa T. and Nagaoka S. (2005). in: J. Cazes (Ed.), Encyclopedia of Chromatography, Taylor \& Francis, London, p. 1528.

Ihara H., Tanaka H., Nagaoka S., Sakaki S. and Hirayama C. (1996). J. Liq. Chromatogr. 19: 2967.

Jinno K., Nagoshi T., Tanaka N., Okamoto M., Fetzer J. C . and Biggs W. R. (1987). J. Chromatogr. 392: 75-82.

Jinno K., Yamamoto K., Ueda T., Nagashima H. and Itoh K. (1992). J. Chromatogr. 594: 105-109.

Jinno K., Yamamoto K., Nagashima H., Ueda T. and Itoh K. (1990). J. Chromatogr. 517: 193.

Jinno K., Ibuki T., Tanaka N., Okamoto M., Fretzer J. C. Biggs W. R., Griffiths P. R. and Olinger J. M. (1989). J. Chromatogr. 461: 209.

Kirkland J. J., Glajch J. L. and Farlee R. D. (1989). Anal. Chem.61: 2-11.

Rahman M. M., Takafuji M., Hamid R. Ansarian, Derakhshan M. and Ihara H. (2005). Anal. Chem., 77 : 6671-6681

Saito Y., Jinno K. and Pesek J. J. (1994). Chromatographia 38: $295-303$

Sakaki S., Kato K., Miyazaki T., Ohkubo K., Ihara H. and Hirayama C. (1993). J. Chem. Soc. Faraday Trans 2: 659.

Shundo A., Sakurai T., Takafuji M., Nagaoka S. and Ihara H. (2005). J. Chromatogr. A 1073: 169.

Takafuji M., Ishiodori A., Yamada T., Sakurai and Ihara H. (2004). Chem com. 24: 1122-1123.

Received: October 10, 2010;

Accepted : January 10, 2011 